\title{
Saúde e marginalização social: suprimindo falhas curriculares
}

\section{Health and social marginalization: suppressing curricular failures}

\section{Salud y marginación social: suprimiendo fallas curriculares}

\author{
Mariana Franco Ribeiro de Oliveira ${ }^{1}$, João Lucas Cruz Castanho², Rodrigo Santos Custódio Oliveira² \\ ${ }^{1}$ Universidade Positivo; GTs de Mulheres e Sexualidade e Diversidade, da SBMFC. Curitiba, PR, Brasil. \\ ${ }^{2}$ Estudante do $6^{\circ}$ ano, da Universidade Positivo. Curitiba, PR, Brasil.
}

\section{Resumo}

Objetivos: Ampliar a compreensão dos estudantes da saúde em relação à dinâmica social que leva à marginalização, suas particularidades clínicas e políticas públicas existentes, discutindo qual o seu papel na mudança da realidade exposta. Método: Realizou-se um estudo descritivo, do tipo relato de experiência, tendo como foco central o Curso de extensão "Saúde e Marginalização Social: expandindo perspectivas" de uma Universidade de Curitiba. O curso, com duração anual, apresentava-se com encontros mensais para discussão dos temas: o sentido da saúde e a influência da marginalização social, saúde das populações negra, indígena, LGB e TRANS, profissionais do sexo, em situação de rua e saúde mental. Em espaços organizados por alunos do curso de Medicina e supervisão docente, os convidados, pertencentes às populações em discussão, ou envolvidos em políticas públicas, demonstraram suas visões e sofrimentos e se relacionaram com a plateia, por meio de recortes históricos, relatos de vivência e descrição das dificuldades e perspectivas dentro da medicina. Resultados: Os espaços apresentaram grande adesão de estudantes, de variados cursos; houve relatos de surpresa e a afirmação de que nunca haviam presenciado esses temas em sala de aula. A maioria relatou elucidação nestas questões e a possibilidade de aplicação clínica dos conteúdos para humanização das suas práticas em saúde. Conclusão: Faz-se necessária a inclusão do tema Populações Marginalizadas nas ementas dos cursos da saúde, a fim de que as discussões representem mudanças no perfil do médico formado, seguindo as competências definidas nas DCNs e no cuidado, agora integral, ofertado aos pacientes.

Palavras-chave: Marginalização Social; Educação Médica; Humanização da Assistência

Como citar: Oliveira MFR, Castanho JLC, Oliveira RSC. Saúde e marginalização social: suprimindo falhas curriculares. Rev Bras Med Fam Comunidade. 2019;14(41):1793. https://doi.org/10.5712/rbmfc14(41)1793
Autor correspondente: Mariana Franco Ribeiro de Oliveira. E-mail: marianafr.oliveira@gmail.com Fonte de financiamento: declaram não haver.

Parecer CEP: não se aplica.

Procedência e revisão por pares: revisado por pares. Recebido em: 25/03/2019. Aprovado em: 03/07/2019. 


\begin{abstract}
Objectives: To increase the health student's understanding regarding social dynamics that leads to marginalization, its clinical features and existing public policies, discussing their role about the change of the exposed reality. Methods: A descriptive study has been made about the type experience report, having as main focus the Extension Program "Health and Social Marginalization: suppressing curricular failures" from a University of Curitiba. The annual program was presented with monthly meetings to discuss the themes: the meaning of health and the influence of the social marginalization, health of the afro descendants, indigenous, LGB and Transsexual populations, sex workers, homeless and mental health. In places organized by medical students with teacher's supervision, the guests, belonging to the populations under discussion or involved in public policy, demonstrated their visions and sufferings getting involved with the audience through history fragments, experience reports and difficulties descriptions and perspectives within medicine. Results: The places presented great adhesion of students from several courses, there were surprised reactions and confirmation that they never had contact with this subjects in class. Most reported elucidation in these questions and the possibility of clinical application of the content to humanize their health care practices. Conclusion: The inclusion of the theme of Marginalized Populations in the health courses programs is necessary to encourage discussions that can make significant changes in the trained physician profile, following the competencies defined in the DCNs and now in the integral care offered to patients.
\end{abstract}

Keywords: Social Marginalization; Education, Medical; Humanization of Assistance

\title{
Resumen
}

Objetivos: Ampliar la comprensión de los estudiantes de la salud con relación a la dinámica social que lleva a la marginación, sus particularidades clínicas y políticas públicas existentes, discutiendo cuál es su papel en el cambio de la realidad expuesta. Método: Se realizó un estudio descriptivo, del tipo relato de experiencia, teniendo como enfoque central el Curso de extensión "Salud y Marginalización Social: expandiendo perspectivas" de una Universidad de Curitiba. El curso, con duración anual, se presentaba con encuentros mensuales para discusión de los temas: el sentido de la salud y la influencia de la marginación social, Salud de las poblaciones negra, indígena, LGB y TRANS, profesionales del sexo, en situación de calle y salud mental. En espacios organizados por alumnos del curso de Medicina y supervisión docente, los invitados, pertenecientes a las poblaciones en discusión, o involucrados en políticas públicas, demostraron sus visiones y sufrimientos y se relacionaron con la audiencia, por medio de recortes históricos, relatos de vivencia y descripción de las dificultades y perspectivas dentro de la medicina. Resultados: Los espacios presentaron gran adhesión de estudiantes, de variados cursos; hubo informes de sorpresa y la afirmación de que nunca habían presenciado estos temas en el aula. La mayoría relató elucidación en estas cuestiones y la posibilidad de aplicación clínica de los contenidos para humanización de sus prácticas en salud. Conclusión: Se hace necesaria la inclusión del tema Poblaciones Marginadas en los programas de los cursos de la salud, a fin de que las discusiones representen cambios en el perfil del médico formado, siguiendo las competencias definidas en las DCNs y en el cuidado, ahora integral, ofrecido a los pacientes.

Palabras clave: Marginación Social; Educación Médica; Humanización de la Atención

\section{Introdução}

O Brasil, desde a sua origem, é um país marcado por forte injustiça social. Dessas desigualdades, surge o processo de exclusão e marginalização social de populações historicamente fragilizadas. Hoje, apesar de uma Constituição democrática, muitos brasileiros ainda não possuem seus direitos assegurados, vítimas dessa brutal exclusão.

No campo da educação e da saúde, podemos perceber características evolutivas que também se distanciam do tema - marginalização social, no momento em que o Modelo Biomédico de Atenção, desenvolvido por anos nos cursos de medicina, carrega o crescimento tecnológico, a superespecialização e o estudo hospitalocêntrico e se afasta das relações médico-pacientes, do olhar integral ao ser humano, da humanização. ${ }^{1,2}$

Nesse cenário nada satisfatório, esse trabalho é uma consequência do questionamento, dos pesquisadores, sobre o nível de compreensão por parte do estudante de medicina em relação às populações que sofrem marginalização social.

De acordo com uma pesquisa baseada no questionário socioeconômico do ENADE, o perfil socioeconômico do estudante de medicina brasileiro, apesar de ações afirmativas governamentais, ainda 
é elitizado e de maioria branca, tendo a maioria renda familiar entre 10 e 30 salários mínimos. Esses dados fazem refletir quais os espaços sociais que o acadêmico de medicina frequenta e para qual realidade suas expectativas estão direcionadas. Além disso, evidencia que os alunos de medicina não representam a maior parte da população brasileira, muitas vezes desconhecendo as especificidades culturais, sociais e econômicas que os diferencia. , $^{3,4}$

Debater sobre minorias sociais, principalmente na qualidade e no acesso à saúde, requer uma compreensão mais profunda deste termo: não são minorias no sentido quantitativo, mas minorias no acesso às estruturas sociais de poder e na sua representatividade. Exemplificando essas minorias, dados de 2014 do Instituto Brasileiro de Geografia e Estatística (IBGE) demonstram que 53\% do total de brasileiros são pretos e pardos, por exemplo. Assim sendo, essa população não é minoria quantitativa em nosso país. Entretanto, representa $65 \%$ da população pobre e mais de $70 \%$ da população muito pobre. E mais, entre os assassinatos cometidos, $70 \%$ são contra a população negra. ${ }^{5,6}$

Além de negros e pardos, as populações marginalizadas compreendem LGBTs, povos indígenas, quilombolas, pessoas privadas de liberdade, pessoas em situações de rua, pessoas com condições psiquiátricas, deficientes físicos, população rural, profissionais do sexo, dentre outras. Populações, essas, que se encontram fragilizadas e apresentam um contraste de vida bastante acentuado, quando comparado à realidade da maior parte dos estudantes de medicina.

Cada população marginalizada atende por uma demanda específica, sendo necessário o básico conhecimento sobre sua real situação social para que ações eficazes, com base humanitária, sejam efetuadas. E dentre essas ações está a prática médica. Quando não nos abrimos ao debate sobre essas especificidades da saúde, soa um alerta: medicina para quê e para quem? O princípio da equidade, em lei pelo Sistema Único de Saúde, afirma que grupos populacionais com necessidades específicas sejam atendidos de forma diferenciada. E, assim, cabe a pergunta: estará o estudante de medicina, após a sua formação, apto a reconhecer tais diferenças entre seus pacientes e, mais que isso, tratá-los com equidade ${ }^{7,8}$

O modelo biomédico de prática da medicina, hegemônico no diagnóstico e tratamento de doenças desde meados do século XIX, aplica uma lógica mecanicista com referência técnico-instrumental nas biociências. Exclui o contexto psicossocial e espiritual do indivíduo e as significações biológicas dentro das subjetividades, dos quais depende uma compreensão plena para que seja feita uma intervenção adequada.

O modelo flexneriano de ensino médico, fortemente incentivado no Brasil no período do regime militar, contribuiu ainda mais para a sedimentação de um currículo biologicista, com viés individualista e com destaque na patologia. Esses modelos impostos, apesar de recente problematização e tentativas de superação, são historicamente hegemônicos dentro das universidades e mantêm suas características, mesmo em cursos ditos de vanguarda, contribuindo para a perpetuação de uma medicina desumanizada. Mudar esse panorama requer uma atitude conjunta entre estruturas administrativas, corpo docente e discente. .,2,9-11 $^{-10}$

Levar debates para dentro da universidade, que dificilmente se instalam dentro das salas de aula, propõe somar conhecimento à academia, contribuindo para a proposta dos cursos de medicina, em relação ao avanço tecnológico e científico incorporado ao ensino; além de se enquadrar nas Diretrizes Curriculares Nacionais do Curso de Medicina de 2014, que refere, dentre inúmeros outros, no item VII 
do Art. 23: "abordagem de temas transversais, no currículo, que envolvam conhecimentos, vivências e reflexões sistematizadas acerca dos direitos humanos e de pessoas com deficiência, educação ambiental, ensino de Libras (Língua Brasileira de Sinais), educação das relações étnico-raciais e história da cultura afro-brasileira e indígena". ${ }^{12}$

Dessa forma, os novos médicos podem estar aptos para o exercício da boa prática médica, e a recuperação do humanismo da profissão, para que o exercício profissional se dê ancorado nas necessidades das pessoas e da sociedade..$^{10,11,13}$

\section{Métodos}

O presente estudo define-se como estudo descritivo, tipo relato de experiência. A coleta de informações para a construção deste relato foi realizada, pelos autores, através da construção, vivência e análise do curso de extensão "Saúde e marginalização social: expandindo perspectivas", em uma universidade particular de Curitiba, durante o período de dois anos (2016 e 2017) em que o curso ocorreu.

O curso teve como objetivo geral fomentar o debate e trazer a presença de representantes de populações negligenciadas nos cursos de saúde, a fim de expandir a visão do acadêmico para um panorama fora de sua realidade, com compreensão da dinâmica social que leva à marginalização.

Adicionado a esse, citamos como objetivos específicos do curso: entender o conceito de marginalização social; discutir os motivos que tornam uma população negligenciada ou em situação de vulnerabilidade; conhecer as relações sociais que caracterizam os grupos estudados; conhecer as particularidades clínicas de cada grupo populacional com base epidemiológica e questionar como esses dados são alcançados e se eles refletem mesmo a realidade do país; discutir quais são as políticas públicas nacionais, regionais e locais que envolvem os grupos em questão, sua real abrangência e o motivo de não funcionarem da maneira como são projetados; discutir de que forma os estudantes de medicina e os profissionais médicos podem interagir com a realidade exposta.

Para a construção dos espaços, optou-se por uma adaptação do método recepcional para ensino de literatura, de Bordini e Aguiar, demostrando o papel preponderante do estudante que vai imprimir valor nas falas expostas, conforme sua bagagem cultural; de modo que, quando o estudante significar o tema, terá mais condições para nele atuar e modificar. ${ }^{14}$

O método consistiu, sucintamente, em abordar, inicialmente, uma situação que se enquadrasse no horizonte de expectativas do estudante: a primeira fala e etapa foi direcionada para o entendimento de particularidades biomédicas do grupo estudado (tema de real interesse para estudantes da área da saúde); a segunda etapa foi a quebra do horizonte de expectativas, aplicando-se uma comparação entre o familiar e o novo, trazido pelo relato de um representante da população minoritária em discussão, sendo profissional de saúde, que atua com a população em questão, e pode trazer contribuições reais dos atendimentos realizados, ou um representante próprio do grupo minoritário, contribuindo com experiências próprias de sua realidade de vida. ${ }^{14}$

Dessa forma, as discussões expandem o horizonte de expectativas dos estudantes, permitindo uma perspectiva mais humanizada e consciente quando se trata do atendimento de um paciente representante de uma população marginalizada socialmente, ou em condição de vulnerabilidade social. ${ }^{10,11,13}$ 
Das atribuições dos mediadores do curso, além de organizar o espaço e controlar o tempo, estava a exposição de informações acerca de políticas públicas idealizadas e implementadas em relação ao grupo discutido e a utilização de músicas, imagens, vídeos e textos, para incorporar um conteúdo mais robusto e dinâmico.

\section{Resultados}

Discussões mensais, que ocorreram sob a forma de painéis, traziam na primeira hora uma exposição do tema, em que um ou mais palestrantes discorreram sobre determinado eixo temático, e segunda e terceira horas destinadas às intervenções, considerações e perguntas da plenária.

As falas foram feitas por pessoas com experiência no assunto tratado no encontro. Após as explanações, o debate entra como uma incitação a refletir as mudanças no horizonte de expectativas dos

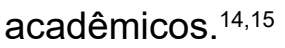

Foi disponibilizado para os participantes um material impresso de cada assunto, contendo dois textos bases: o primeiro de cunho biológico/epidemiológico e o segundo de cunho social/político. Além disso, o material contou com uma seção cultural, reunindo relações literárias, musicais, cinematográficas, dentre outras, que abordavam o tema em questão e uma seção bibliográfica, que estendeu as discussões e elucidações sobre o assunto.

Temas abordados

Saúde da População Negra

Saúde da População Indígena/populações tradicionais (ciganos, quilombolas, comunidade de curandeiras, colônias europeias)

Saúde da População Rural

Saúde da População em situação de rua

Saúde da População LGBT

Pacientes psiquiátricos e Luta Antimanicomial

Saúde Mental do Estudante de Medicina

Saúde da População Carcerária

Saúde de Profissionais do Sexo

Vítimas de Violência e Opressão

Os encontros, que foram organizados e oferecidos como Cursos de Extensão Universitária a estudantes da área da saúde, reuniram um número variável de estudantes de diferentes cursos, chegando a um máximo de 80 pessoas. Em média, participaram 45 estudantes por tema, sendo em sua maioria estudantes da área da saúde, principalmente da medicina.

Uma característica importante observada foi a presença e participação de estudantes que já apresentavam interesse no assunto, seja pela participação em ligas relacionadas ao tema, em palestras e congressos, nos centros acadêmicos, dentre outros.

Se mantém a pergunta de como alcançar um público diferenciado de estudantes, que é quem realmente necessita dessa abordagem e treinamento. Uma das alternativas é a inserção do tema na ementa do curso de medicina, na grade curricular, corroborando com as solicitações das DCN's. ${ }^{1,12}$ 
Os estudantes, ao final dos encontros e nas conversas "de corredor", demonstraram muita animação nas discussões e temas e a vontade de ampliar o conhecimento de mais formas. Também exibiam a vontade e necessidade de treinamento prático das habilidades de atendimento às populações. Contavam que jamais tiveram aulas semelhantes nos seus cursos e que os encontros trouxeram um grande crescimento pessoal.

\section{Discussão}

Nos encontros realizados nos horários de contra turno, à noite, após atividades acadêmicas obrigatórias, com duração de cerca de três horas, observou-se grande dificuldade em motivar a participação, tendo em vista que a carga horária do curso de medicina é muito grande e os estudantes, em geral, estão sobrecarregados. Sob a mesma justificativa, houve dificuldades na organização e divulgação mais efetiva.

Uma questão bastante relevante sobre o título do trabalho fora levantada durante a execução do projeto: o termo marginal/marginalidade/marginalização seria adequado para se referir às populações abordadas? Levando em consideração um mecanismo estrutural de manutenção das opressões, chegamos ao ponto comum entre as pessoas consideradas marginalizadas e pessoas consideradas oprimidas.

Apesar de os conceitos terem origens diferentes, esse político e aquele econômico, as pessoas oprimidas não vivem fora da sociedade e, portanto, não são marginais a ela.

Em uma sociedade de classes, o papel do oprimido é manter o mecanismo de exploração do trabalho em funcionamento pleno, dessa forma, levamos a discussão para um processo de adoecimento cujo determinante é, essencialmente, social. ${ }^{15,16}$

Em um contexto capitalista, as pessoas marginalizadas são, em geral, as de menor poder aquisitivo e, no Brasil, segundo o IBGE, 10\% da população concentram quase metade da renda do país. A concentração de renda evidencia, então, que os pobres, como já colocado antes, são explorados economicamente, e tal fato é importante para manutenção desse processo..$^{15-17}$

As políticas públicas específicas para as populações, já citadas nesse trabalho, são construções coletivas que visam promover a equidade nos serviços de seguridade social. Embora esses direitos sejam garantidos por leis, constitucionalmente, ainda é necessária a fundamentação de documentos para regularização desses serviços e ampliação do acesso das populações, às políticas citadas. ${ }^{15,16}$

Levando em consideração o perfil do estudante de medicina, majoritariamente branco, de classe média, é importante ter representatividade no momento em que trabalhamos o contexto das populações marginalizadas. ${ }^{15-17}$

Organizar um espaço no meio acadêmico que dê voz a representantes das populações marginalizadas é, também, um ato político de resistência, além de todas as questões educacionais já discutidas e implementadas nas Diretrizes Nacionais Curriculares dos cursos de medicina, principalmente em sua revisão de 2014. ${ }^{12,15,16}$

Essa discussão, em torno da inserção de temas relevantes na universidade, deve ser permeada pelo histórico evolutivo da educação médica, que culminou com o distanciamento nas relações médico-paciente, que, percebido pela sociedade, resultou em descrédito nas práticas médicas atuais. O modelo biomédico, citado anteriormente, demonstra-se insuficiente em acompanhar o sofrimento, as necessidades emocionais dos pacientes e suas implicações subjetivas, sendo urgentes mudanças nesse sentido. ${ }^{18-23}$ 
Em quase 30 anos de evolução do ensino médico, no Brasil, algumas mudanças trouxeram novos horizontes à realidade até então observada: a promulgação da nova Constituição e a criação do Sistema Único de Saúde, em 1990; as mudanças das Diretrizes Curriculares Nacionais, em 2001 e sua revisão, em 2014; e a criação da Política Nacional de Humanização com o programa Humaniza SUS, em 2003. ${ }^{18-21}$

A promulgação da nova Constituição, em 1988, trouxe um maior enfoque no ser humano, e carregou as mudanças no campo da saúde, com a posterior criação da Lei 8080 - Lei Orgânica da Saúde, em que temas como Integralidade do cuidado e humanização do atendimento entram em foco e começam a modificar os parâmetros educacionais exigidos até aquele momento. ${ }^{18-21}$

Baseado, também, nessas preocupações, o Conselho Nacional de Educação, em 2001 e em 2014, aprovou as Diretrizes Curriculares Nacionais (DCN) da Medicina e trouxe enfoque ao tema humanização, com todas as suas particularidades e a inserção da discussão sobre o indivíduo biopsicossocial-espiritual, suas relações com a sociedade e seu processo de negligenciação, como exigência nas práticas dos cursos e como habilidade indispensável à formação do profissional médico. As competências humanizadas são saberes que podem e devem ser ensinados, não dependendo apenas do modo de criação e personalidade de cada pessoa, e devem ser pautadas na aquisição de conhecimentos de domínio técnico, ético e relacional, para que se alcance maior efetividade nos atendimentos à saúde.1,12,18,20

Nesse período, os cursos de medicina têm tentado reestruturar seus Projetos Pedagógicos, mas ainda não apresentam uma resposta, em relação às metodologias mais corretas a serem utilizadas, para alcançar os objetivos propostos, somado à dificuldade nos aspectos subjetivos da construção da identidade do profissional médico, que ressalta a competência isolada do profissional, dificultando as experiências intersubjetivas. A proposta atual, para essas mudanças, pauta-se na transformação do ensino da medicina mais focado na mudança social — de natureza ético-política - a partir de novas práticas transformadoras da cultura de formação médica. ${ }^{18,21-23}$

Enfim, para que a transformação do olhar e das práticas profissionais da saúde ocorra, no futuro, é necessário que todos os envolvidos no processo de ensino-aprendizagem - a IES, a gestão local do sistema de saúde, os trabalhadores da saúde, os docentes e os próprios estudantes - tenham compreensão do seu papel profissional nessa mudança cultural e que novos métodos de ensino sejam testados e inseridos no currículo médico. ${ }^{18,20,22,23}$

\section{Conclusão}

O curso reuniu, durante os dois anos de execução, uma quantidade relevante de estudantes, vivenciando discussões distintas de suas realidades e que não estão presentes no dia-a-dia curricular de seus cursos universitários. Sendo assim, desenvolveu discussões teóricas intensas, de cunho social, político, econômico, ambiental, dentre outros, que fomentaram o sentimento de humanização nos estudantes, demonstrado posteriormente em suas falas e práticas na universidade.

Apesar disso, por ser parte de um currículo paralelo não obrigatório, o curso agregou, em sua maioria, estudantes que já tinham interesse ou vivências com os temas explorados e nos demonstrou a necessidade de buscar novos meios de inserção do tema para um número maior de estudantes. 
Sendo o tema, segundo a revisão exposta, tão importante para a formação médica de qualidade, podemos entender a necessidade de implementá-lo como parte do currículo obrigatório, nos planos pedagógicos dos cursos de medicina, a fim de que se alcance o desenvolvimento profissional almejado e a qualidade de cuidado oferecido às populações oprimidas.

Além disso, práticas de extensão universitária reais, com treinamento externo das habilidades dos estudantes e convivência com as populações em estudo, seriam de grande relevância no aumento da qualidade das discussões e do treinamento dos estudantes.

Falar de saúde é abordar a medicina no seu propósito fundamental. Falar de populações marginalizadas é dar voz àqueles que não têm vez.

\section{Contribuição dos autores}

Declaramos que participamos de forma suficiente na concepção e desenho deste estudo ou da análise e interpretação dos dados assim como da redação deste texto, para assumir a autoria e a responsabilidade pública pelo conteúdo deste artigo. Revisamos a versão final deste artigo e o aprovamos para ser encaminhado à publicação. Declaramos que nem o presente trabalho nem outro com conteúdo substancialmente semelhante de nossa autoria foi publicado ou submetido à apreciação do Conselho Editorial de outra revista.

\section{Agradecimentos}

Em tempo, agradecemos a algumas instituições que foram essenciais para a realização do projeto que originou o trabalho apresentado, tal qual a Direção Executiva Nacional dos Estudantes de Medicina, ao Transgrupo Marcela Prado, à equipe do Consultório de Rua, e à professora Mariana, aqui colocada como autora, por ter apoiado a essa ideia prontamente e por ter investido no processo de construção desse projeto tão importante para nossa reafirmação enquanto estudantes e profissionais da saúde.

\section{Conflito de interesses}

Declaram não haver.

\section{Referências}

1. Streit DS, Barbosa Neto F, Lampert JB, Lemos JMC, Batista NA. 10 anos de Diretrizes Curriculares Nacionais. Rio de Janeiro: ABEM; 2012.

2. Gomes AP, Costa JRB, Junqueira TS, Arcuri MB, Siqueira-Batista R. Atenção Primária à Saúde e Formação Médica: entre Episteme e Práxis. Rev Bras Educ Med. 2012;36(4):541-9. DOI: https://doi.org/10.1590/S0100-55022012000600014

3. Oliveira NA, Alves LA. Ensino Médico, SUS e início da profissão: como se sente quem está se formando. Rev Bras Educ Med. 2011;35(1):26-36. DOI: https://doi.org/10.1590/S0100-55022011000100005

4. Ristoff D. Perfil socioeconômico do estudante de graduação: uma análise de dois ciclos completos do Enade (2004 a 2009). Cad GEA. 2013;2(4):2-32.

5. Brasil. Instituto Brasileiro de Geografia e Estatística (IBGE). Síntese de indicadores sociais: uma análise das condições de vida da população brasileira. Rio de Janeiro: IBGE; 2013. 
6. Brasil. Instituto Brasileiro de Geografia e Estatística (IBGE). A janela para olhar o país. PNAD - Pesquisa Nacional por Amostra de Domicílios. Rio de Janeiro: IBGE;2015 [Acesso 2018 Fev 17]. Disponível em: http://www.ibge.gov.br/home/presidencia/noticias/imprensa/ ppts/00000024052411102015241013178959.pdf

7. Gouveia R, Palma JJ. SUS: na contramão do neoliberalismo e da exclusão social. Estud Av. 1999;13(35):139-46. DOI: https://doi. org/10.1590/S0103-40141999000100014

8. Lucchese PTR. Equidade na gestão descentralizada do SUS: desafios para a redução de desigualdades em saúde. Ciênc Saúde Coletiva. 2003;8(2):439-48. DOI: https://doi.org/10.1590/S1413-81232003000200009

9. Laurell AC. A saúde-doença como processo social. Tradução E. D. Nunes. Rev Latinoam Salud. 1982;2:7-25.

10. De Marco MA. Do modelo biomédico ao modelo biopsicossocial: um projeto de educação permanente. Rev Bras Educ Med. 2006;30(1):60-72. DOI: https://doi.org/10.1590/S0100-55022006000100010

11.Pagliosa FL, Da Ros MA. O relatório Flexner:para o bem e para o mal. Rev Bras Educ Med.2008;32(4):492-9. DOI: https://doi.org/10.1590/ S0100-55022008000400012

12. Brasil. Ministério da Educação. Diretrizes Curriculares Nacionais dos Cursos de Graduação em Medicina. Brasília: Ministério da Educação; 2014 [acesso 2018 Mar 23]. Disponível em: http://portal.mec.gov.br/index.php?option=com_docman\&view=download\&alias=15874rces003-14\&ltemid=30192

13. Bordini GM, Aguiar VT. A Formação do Leitor: alternativas metodológicas. Porto Alegre: Mercado Aberto;1988. p. 81-99.

14. Manosso PZ. Contribuições do Método Recepcional para a Formação de Leitores e Alunos-Autores no Ensino Fundamental I [Dissertação]. Curitiba: Universidade Federal do Paraná; 2017.

15. Santos M. Pobreza Urbana. 3ª ed. São Paulo: Editora da Universidade de São Paulo; 2013.

16. Brasil. Instituto Brasileiro de Geografia e Estatística (IBGE). 10\% da população concentram quase metade da renda do país [acesso 2018 Jun 28]. Disponível em: https://agenciadenoticias.ibge.gov.br/agencia-noticias/2012-agencia-de-noticias/noticias/20844-10-dapopulacao-concentram-quase-metade-da-renda-do-pais

17. Barros JAC. Pensando o processo saúde doença: a que responde o modelo biomédico? Saude Soc. 2002;11(1):67-84.

18. Rios IC, Schraiber LB. Humanização e Humanidades em Medicina: a formação médica na cultura contemporânea. 1ํㅡㄹ ed. São Paulo: Editora UNESP; 2012.

19. Marins JJN, Rego S, Lampert JB, de Araújo JGC, orgs. Educação Médica em Transformação: instrumento para construção de novas realidades. São Paulo: Hucitec; 2004.

20. Feuerwerk LCM. Além do discurso de mudança na educação médica: processos e resultados. São Paulo: Hucitec; 2002.

21. Hortale VA, Moreira COF, Bodstein RCA, Ramos CL, orgs. Pesquisa em saúde coletiva: fronteiras, objetos e métodos. Rio de Janeiro: Editora Fiocruz; 2010.

22. Nunes ED. Saúde coletiva: história de uma idéia e de um conceito. Saude Soc. 1994;3(2):5-21. DOI: https://doi.org/10.1590/S010412901994000200002

23. Rios IC. Caminhos da humanização na saúde - prática e reflexão. São Paulo: Áurea Editora; 2009. 